\title{
ACOUSTIC SPECTROSCOPY OF NANOSTRUCTURES IN CONDENSED MATTER
}

\author{
Peter BURY \\ Department of Physics, Faculty of Electrical Engineering, University of Žilina, Univerzitná 1, 01026 Žilina, Slovak Republic, \\ tel.: +421 41513 2300, e-mail: peter.bury@uniza.sk
}

\begin{abstract}
The methods of acoustic spectroscopy are powerful tool for the study of physical properties of condensed matter containing nanostructures. The ability of these methods makes it possible to obtain important information for variety of materials concerning not only their mechanical properties. The principles of acoustic spectroscopy of some kinds of materials and structures as results of a interaction between the acoustic wave and charge curriers and/or nanoparticles are presented. Semiconductor structures and magnetic fluids are the representative selection of condensed matter manifested the convenience of acoustic spectroscopy methods to obtain some physical parameters of nanostructures.
\end{abstract}

Keywords: acoustic spectroscopy, semiconductor structures, magnetic fluids

\section{INTRODUCTION}

The physical acoustics plays important role in the continuing development of our understanding of the physics of solids. Atoms that have combined to form a crystalline or amorphous solid constitute a medium capable of propagating all kinds of mechanical vibrationsacoustic waves some of them can also propagate through liquids. If they have wavelengths much larger than the sizes and spacing of the atoms acoustic waves propagates as in continuous medium. In addition to frequency, the characteristic parameters of these waves include phase velocity and attenuation. By measuring these parameters as a function of temperature, frequency, electric or magnetic field, illumination representing the methods of acoustic spectroscopy a great variety of information about the physical properties of the material under investigation can be obtained [1].

In this contribution we present mainly the principle of acoustic spectroscopy for the investigation of some kinds of materials with nanostructures and/or nanoparticles as a result of the interaction between acoustic wave and charge curriers at interface of semiconductor structures or magnetic particles in magnetic fluids. The results obtained on both the MOS (metal-oxide-semiconductor) structures with ultra-thin oxides prepared by NAOS (nitric acid oxidation) technology and on the transformer oils based magnetic fluids to verify the method of acoustic spectroscopy are presented and discussed.

\section{ACOUSTIC SPECTROSCOPY OF INTERFACE STATES IN MOS STRUCTURES WITH ULTRA- THIN OXIDES}

\subsection{Theoretical principles}

Interface states at semiconductor-insulator heterojunction in MOS structures play an important role in determining their electrical characteristics that are important for practical use in semiconductor devices. A silicon-silicon dioxide $\left(\mathrm{Si} / \mathrm{SiO}_{2}\right)$ structure mostly prepared by thermal oxidation at above $800^{\circ} \mathrm{C}$ in oxidizing atmospheres is widely used for MOS devices [2]. Because the high temperature oxidations results in high interfacial stress producing the interface defect states, the low temperature direct oxidation methods such as plasma oxidation or method of nitric acid oxidation of $\mathrm{Si}$ were developed [3]. Although due to the many improvements in preparation technology the density of interface states, especially at $\mathrm{SiO}_{2} / \mathrm{Si}$ interfaces is usually very low, it can still seriously affect electrical properties of MOS devices formed from these structures.

When the acoustoelectric (AE) effect in semiconductor structures has been shown to be a useful tool for the experimental study of interface deep centres, two basic modifications of acoustic (acoustoelectric) deep-level transient spectroscopy (A-DLTS) were introduced. The surface acoustic wave (SAW) technique uses a nonlinear AE interaction between the SAW electric field and free carriers in an interface region which generates a transverse AE signal (TAS) across the structure [4]. The longitudinal acoustic wave (LAW) technique uses an acoustoelectric response signal observed at the interface of the semiconductor structure when a longitudinal acoustic wave propagates through the structure $[5,6]$.

The LAW, following the pressure modulation of the charge at the semiconductor structure interface, evokes the change of the potential difference that manifests as an acoustoelectric response signal (ARS). The ARS produced at the interface of the MOS structures is very sensitive to any changes in the space charge distribution in the interface region and its dependence on the bias voltage ( $U_{a c}-U_{g}$ curve) can be then used to study the distribution of interface states in MOS structures especially with ultrathin oxide layers.

The interface states at MOS interfaces change their charge state depending on whether they are filled or empty, however, the state occupancy varies with gate voltage $U_{g}$ that changes the band bending $\left(q \varphi_{s}\right)$. The gate voltage $U_{g}$ relates to surface potential $\varphi_{s}$ following the equation

$$
U_{g}=\varphi_{s}-\frac{Q_{s}\left(\varphi_{s}\right)}{C_{o x}}-\frac{Q_{i t}\left(\varphi_{s}\right)}{C_{o x}}+\varphi_{m s}-\frac{Q_{o x}}{C_{o x}},
$$

where $Q_{s}$ is the semiconductor charge, $\Phi_{m s}$ is the work function difference between gate and semiconductor, $Q_{i t}$ is 
the trapped charge at the interface state, $Q_{o x}$ is the oxide charge and $C_{O X}$ is the oxide capacitance. The last two terms in the Eq.(1) represents the flat band voltage $U_{f b}$. The charge trapped into interface states changes with any change in the band bending.

The acoustic wave, following the pressure modulation of charge density evokes the change of the potential difference that manifests as an ARS signal. The ARS produced by a MOS structure propagating by longitudinal acoustic wave can be then expressed using the similarity with the case of electromechanical capacitance transducer of thin planar structure $(d<<\lambda)$ by the relation $[5,7]$

$$
U_{a c}=\phi_{s} \frac{p}{K_{s}}-\frac{Q_{s}\left(\varphi_{s}\right)}{C_{o x}} \frac{p}{K_{i}}-\frac{Q_{i t}\left(\varphi_{s}\right)}{C_{o x}} \frac{p}{K_{i}}
$$

and its amplitude as

$$
U_{a c}^{o}=\left|\phi_{s} \frac{p_{0}}{K_{s}}-\frac{1}{C_{o x}} \frac{p_{0}}{K_{i}}\left[Q_{s}\left(\varphi_{s}\right)+Q_{i t}\left(\varphi_{s}\right)\right]\right|,
$$

where $K_{s}$ and $K_{i}$ are the elastic moduli of the semiconductor and insulator, respectively, $p_{0}$ is the acoustic pressure amplitude. The physical meaning of the absolute value is that the ARS cannot differ the total charge or potential polarity.

If the situation in the structure without any interface states $\left(Q_{i t}=0\right)$ is indexed as “ideal” then using Eq. (1) the last relation can be expressed in the form

$$
U_{a c}^{0}(\text { ideal })=\left|\frac{p_{0}}{K_{s}}\right|\left(U_{g}-U_{f b}\right)\left|+\frac{Q_{s}}{C_{o x}} \frac{K_{i}-K_{s}}{K_{s} K_{i}} p_{0}\right| .
$$

As it can be seen from the Eq. (4) the ARS of ideal MOS structure is the superposition of linear term with zero at flatband voltage and term representing the contribution from the semiconductor charge $Q_{s}$.

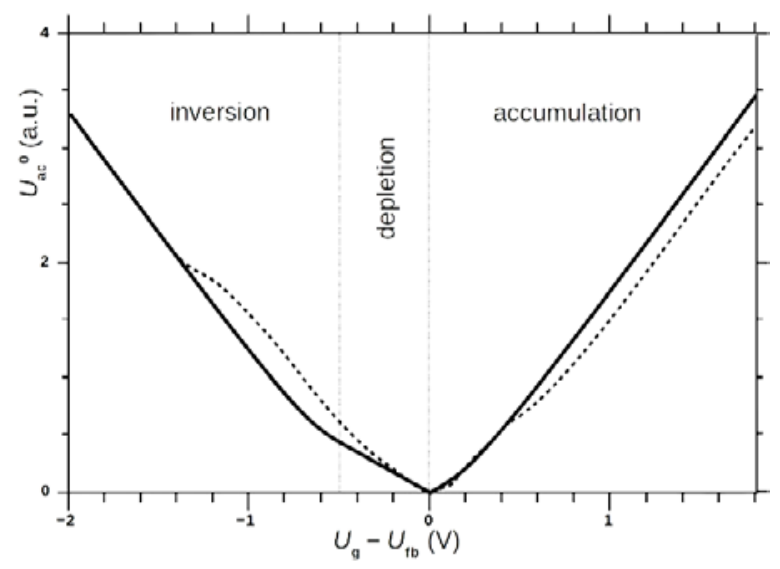

Fig. 1 Theoretical $U_{a c}-U_{g}$ characteristic of "ideal" n-MOS structure-without any interface states (thick line) compared with simulated "real" $U_{a c}-U_{g}$ curve corresponding to two kinds of interface states (thin line)
Comparing Eq.(3) and Eq.(4) the interface trapped charge can be expressed through the deviation of the ARS of real and ideal structures as

$Q_{i t}=S\left(U_{a c}^{0}-U_{a c}^{0}(\right.$ ideal $\left.)\right)$,

where $S=C_{o x} K_{s} / p_{o}$. The interface state density $D_{i t}$ can be then expressed, using the ARS-voltage curves for ideal and real MOS structures, by the relation

$D_{i t}\left(E_{t}\right)=\frac{1}{q}\left|\frac{d Q_{i t}}{d \varphi_{s}}\right|=\frac{1}{q}\left|S \frac{d\left(U_{a c}^{0}-U_{a c}^{0}(\text { ideal })\right)}{d \varphi_{s}}\right|$.

The Eq. (6) allows than to determine the modeling of the distribution of interface states from the ARS.

In the case of real MOS structure with the interface states, the $U_{a c}-U_{g}$ characteristics contains the information about the charge at the interface states. Because the ARS reflects directly changes in the space charge distribution at the interface region, the interface state density can be extracted comparing the ideal (calculated) and real (measured) $U_{a c}-U_{g}$ curves (Eq.6). As the interface state occupancy varies with the gate bias the corresponding changes manifest as hops on the real $U_{a c}-U_{g}$ curve comparing with the ideal one (Fig.1).

However, the leakage current represented by tunnelling transport in the case of very thin oxide layers (< $10 \mathrm{~nm}$ ) becomes a significant problem. The tunnelling current for very thin oxide layers influences the division of the applied voltage $U_{g}$ between the semiconductor and the insulator layer and for the oxide layer thickness $<2$ $\mathrm{nm}$ the whole applied voltage practically spreads across the semiconductor, especially in the range of inversion [8]. Concerning the tunnelling process the transport of free charge curriers through the thin oxide layer caused by electric field has to be taken into account where the tunnelling current can be expressed in the form

$$
J \sim a_{1}\left[\left(U_{g}-U_{f b}\right)^{2}+a_{2}\right]\left[\exp \frac{a_{3}}{U_{g}-U_{f b}}\right],
$$

where $a_{1}, a_{2}, a_{3}$ are constants and the current is supposed to be thermally independent. The simulation of the "ideal" ARS inclusive the calculation of tunnel current contribution gives a new ideal ARS, $U_{a c}^{0}$ (tunnel). The interface charge can be then expressed using the new ideal and real ARS-voltage curves for MOS structure by the relation

$Q_{i t}=S \mid\left(U_{a c}^{0}-U_{a c}^{0}(\right.$ tunnel $\left.)\right) \mid$.

The calculation of $U_{a c}-U_{g}$ curves including the tunnelling process of the free charge curriers through the thin oxide layer changes the "ideal" ARS and gives a new ideal ARS, $U_{a c}^{0}$ (tunnel). Fig.2 illustrates the comparison of $U_{a c}^{0}$ (ideal) $-U_{g}$ and $U_{a c}^{0}$ (tunnel) $-U_{g}$ curves calculated for $3 \mathrm{~nm}$ thick oxide. 


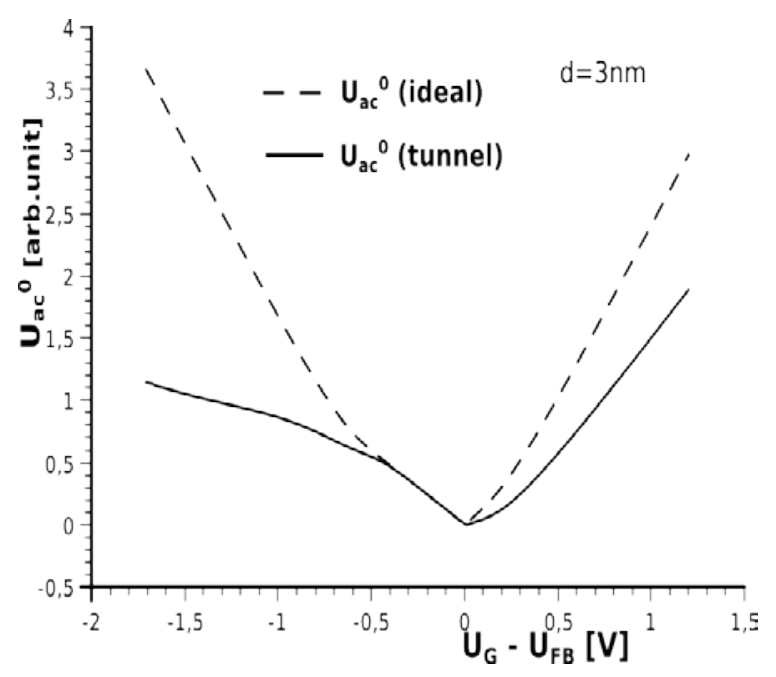

Fig. 2 Theoretical $U_{a c}-U_{g}$ characteristic of "ideal" n-MOS structure without any interface states (dotted line) compared with simulated "ideal" $U_{a c}-U_{g}$ curve for the same structure comprehensive of tunnelling process (full line)

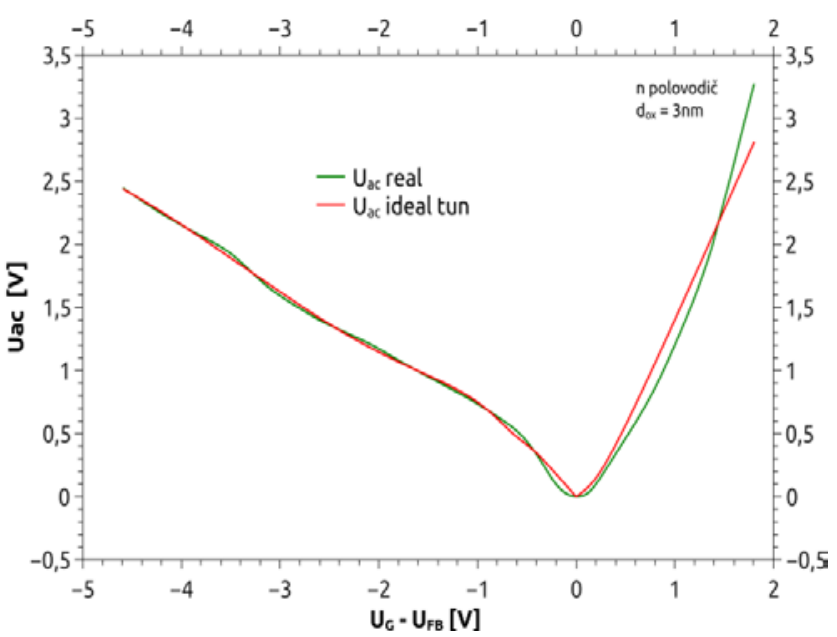

(a)

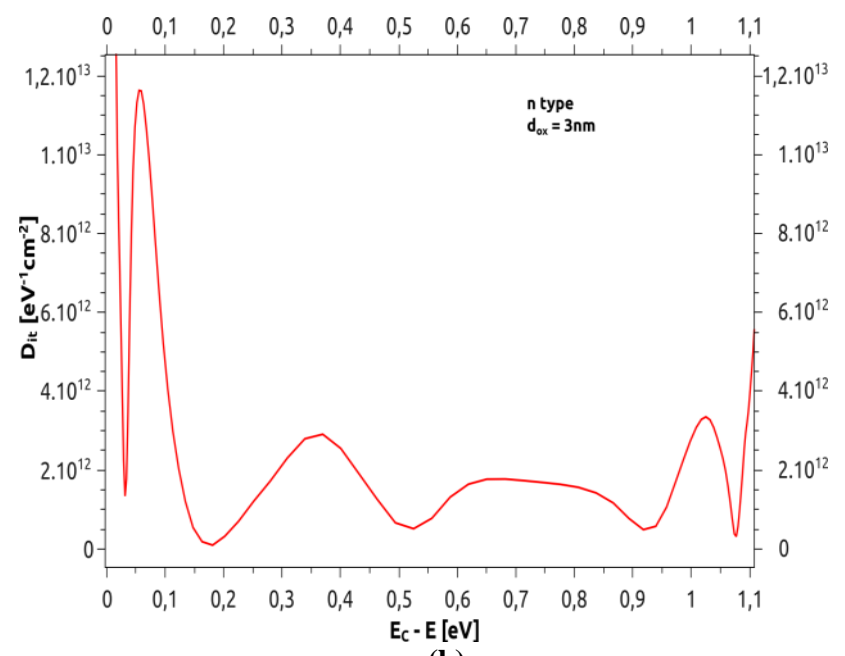

(b)

Fig. 3 Measured and ideal $U_{a c}-U_{g}$ curves (a) and calculated distribution of interface state density (b) for MOS structure $\mathrm{Si}-\mathrm{SiO}_{2}(3 \mathrm{~nm})-\mathrm{Al}$

\subsection{Experimental results}

The interface states were investigated using acoustic spectroscopy in MOS structures prepared on n- type Si (100) wafers with $\sim 10 \Omega \mathrm{cm}$ resistivity with oxides growing by nitric acid $\left(\mathrm{HNO}_{3}\right)$ oxidation method of $\mathrm{Si}$ (NAOS), which can be performed at relatively low temperatures $\left(\sim 120^{\circ} \mathrm{C}\right)$ [9]. The $U_{a c}^{0}-U_{g}$ measurements were performed using the same equipment as for ADLTS technique that has been already described [6,9] but with the voltage source providing the linear increase of gate bias.

Representative results obtained on NAOS Si MOS structure with $3 \mathrm{~nm}$ thick oxide layer is illustrated in Fig. 3. The obtained results coincide very well with the results obtained from the A-DLTS, the spectra of which presented the energy of interface states $0.31-0.40 \mathrm{eV}$ below the conduction band [9].

The density of states distribution using $U_{a c}-U_{g}$ measurement allows us to complete the acoustic investigation of MOS structures represented by the ADLTS and also provides information comparable with $C$ $U_{g}$ and $G-U_{g}$ measurements. The excellent agreements between the results obtained by different acoustic techniques definitely establish the acoustic spectroscopy as a useful tool for the interface states investigation. However, some developments of presented method including both tunnelling process and back side contact is necessary for perfect acoustic characterization of MOS structures with ultrathin oxide layers.

\section{STRUCTURE OF TRANSFORMER OIL-BASED MAGNETIC FLUIDS STUDIED USING ACOUSTIC SPECTROSCOPY}

\subsection{Characterization}

Magnetic fluids (MF) have attracted remarkable physical properties that have recently found wide application in technology $[10,11]$. The transformer oilbased (MF) prepared by adding magnetic nanoparticle suspension to transformer oil with the purpose to improve some of the oil's insulating and thermal properties is an innovating example of the development in power electronic technology. The transformer oil usually used for both high voltage insulation and power electronics cooling is subjected to extensive research to enhance its characteristics [12, 13]. The dielectric breakdown strength of transformer oil, however, is strongly influenced by the aggregation effects of magnetic particles and can induce electric breakdown [14].

For the making improvements in the transformer oilbased ferrofluids, it is important to find the methods the results of which enable to describe the physical behaviour of these liquids, and in particular, the magnetic field induced structure changes of magnetic nanoparticles. One of the useful tools to study changes in the MF structure is based on the measurements of changes in acoustic wave attenuation $\Delta \alpha$ of MF under the influence of an external magnetic field and temperature. The structural changes (the process of clusters formation) in $\mathrm{MF}$ induce additional changes in acoustic attenuation, so that the interaction between the acoustic wave and the aggregated 
magnetic particles or clusters produced in the presence of external magnetic field leads to the additional absorption of acoustic wave $[15,16]$.

The dependence of the attenuation of acoustic wave on the angle $\phi$ between the direction of propagation and that of the magnetic field (anisotropy) provides also important information on the ferrofluid structure in a magnetic field. The comparison of the experimental results with the theoretical predictions $[17,18]$ allows the distinguishing of two motions of the clusters of the ferrous colloidal particles in the fluid (the rotation and translation motions) and the determination of the clusters radius, the density and other parameters of the colloidal particles.

\subsection{Experimental results}

The measurements of the attenuation of the acoustic wave of frequency $5 \mathrm{MHz}$ and $13.3 \mathrm{MHz}$ were carried out by a pulse method using the acoustic spectrometer already described [19]. An acoustic wave generated by $\mathrm{LiNbO}_{3}$ or quartz transducer, propagated through the MF placed in the thermostated closed measuring cell $\left(1.5 \times 0.9 \times 1 \mathrm{~cm}^{3}\right)$ inserted in an electromagnet underwent a multiple reflection between transducers. The temperature was stabilized with an accuracy $\pm 0.2{ }^{\circ} \mathrm{C}$. The first two selected adjacent echoes representing different paths after reflection and reaching the receiving transducer were received by acoustic spectrometer and their ratio represents the acoustic attenuation in MF. They were displayed by the oscilloscope and evaluated and recorded by a computer. Computer also controlled current source for electromagnet.

The dependence of the acoustic wave attenuation on external magnetic field at constant rate of its increase or decrease (2,2 $\mathrm{mT}$ per minute) and constant temperature of MF is shown in Fig. 4. This figure presents the attenuation changes for magnetic field $\boldsymbol{B}$ parallel to $\boldsymbol{k}$, for representative $2.5 \% \mathrm{MF}$ based on transformer oil TECHNOL and $1.25 \%$ MF based on transformer oil ITO 100 , where magnetic field linearly increased to maximum value $100 \mathrm{mT}, 200 \mathrm{mT}$ and $300 \mathrm{mT}$, respectively.

Similar behaviour as in Fig. 4a was observed also for concentrations $0,5 \%, 1,0 \%$ and $2 \%$ of MF based on TECHNOL and result obtained on MF based on ITO 100 for $2,5 \%$ and $5,0 \%$ concentrations were similar to those of Fig. 4b.

The observed results definitely show a strong influence of the magnetic field on the acoustic attenuation of investigated MF based on transformer oils. With increasing magnetic field, the acoustic attenuation from the beginning increases, because the interactions between the magnetic field and the magnetic moment of the nanoparticles lead to aggregation of particles and clusters formation (structures as long as hundreds of nanometers). The behaviour for higher magnetic fields (> $120 \mathrm{mT}$ ) depends on the kind of MF (carrier liquid), concentration of nanoparticles and temperature.

However, the character of attenuation changes with decreasing magnetic field even continues or at least achieved state remains unvaried. This is the reason that the changes of the acoustic attenuation show a hysteresis. This effect can be explained by both the existence of clusters, which lifetimes after their formation are longer than time of decrease of the MF and the process of the continuing formation of clusters also at lower magnetic fields, so that the structure does not return to the initial state immediately after the magnetic field has been removed.
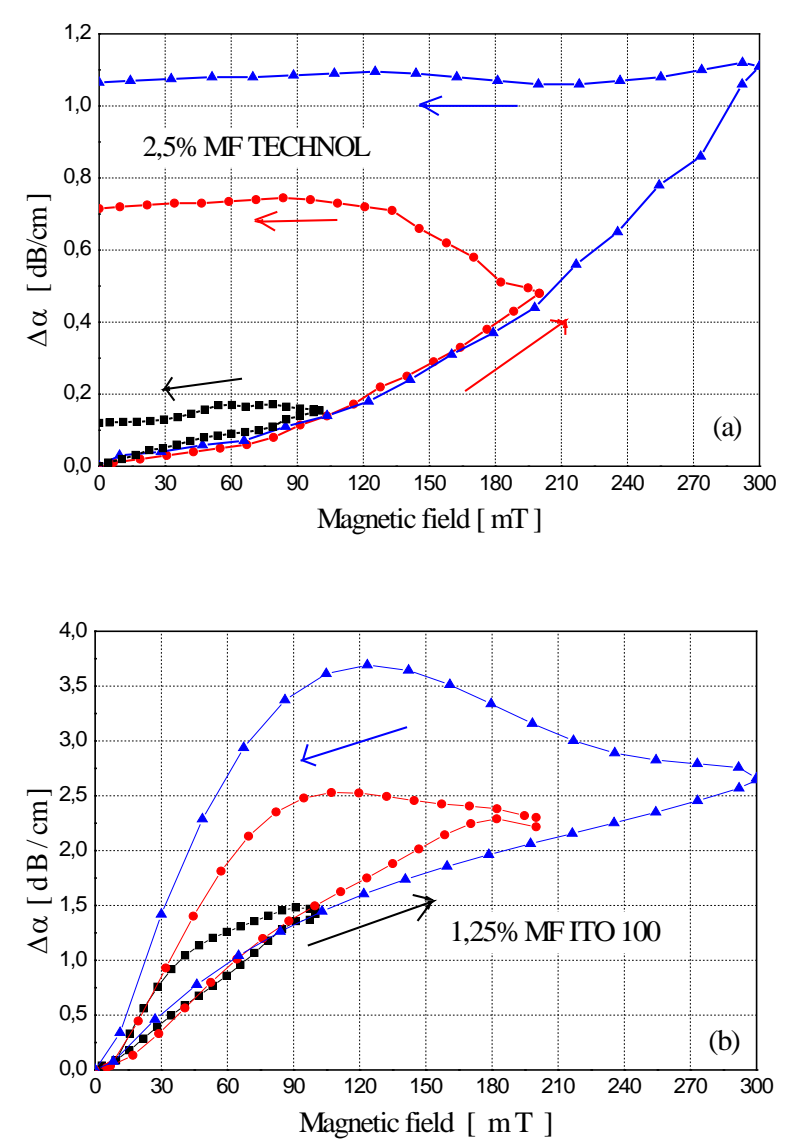

Fig. 4 Changes of the acoustic attenuation in external magnetic field for $2.5 \%$ MF TECHNOL ( $f=13.3 \mathrm{MHz}$ ) (a) and $1.25 \%$ MF ITO 100 ( $f=5 \mathrm{MHz}$ ) (b) measured at $20^{\circ} \mathrm{C}$ for different achieved maximum values of magnetic field ( $\square-100$ $\mathrm{mT}$, • - $200 \mathrm{mT}, \boldsymbol{\Lambda}$ - $300 \mathrm{mT}$ ). Magnetic field increased and/or decreased at constant rate, $2,2 \mathrm{mT} / \mathrm{min}$

Fig. 5 illustrates the representative results of the attenuation anisotropy measured at $20^{\circ} \mathrm{C}$ and magnetic field $200 \mathrm{mT}$ for MF based on TECHNOL (a) and ITO 100 (b). In order to determine the contribution of the acoustic wave energy to the translational and rotational motions the Taketomi functions [18] were used to fit the experimental data:

$$
\begin{aligned}
& \alpha_{\text {rot }}=\frac{2 \pi^{2} f}{\rho_{0} c^{3}}\left(\frac{4}{3} \eta+\eta_{V}+2 \alpha_{5} \cos ^{2} \phi+\alpha_{1} \cos ^{4} \phi\right), \\
& \alpha_{t r}=\frac{3 \pi \eta_{0} a \omega^{3} \rho_{0} V N\left(6 \pi \eta_{0} a+\rho_{0} V \omega\right) /\left(k c^{2}\right)}{\left(\sin \phi-\rho_{m} V \omega^{2} / k\right)^{2}+\left(6 \pi \eta_{0} a \omega / k\right)^{2}},
\end{aligned}
$$

where $c$ is the velocity of the acoustic wave propagating at an frequency $f, \rho$ is the density of the carrier fluid, $\eta$ and $\eta_{\mathrm{V}}$ are the dynamic and volume viscosities of the ferrofluid and $\alpha_{1}, \alpha_{5}$ are the Leslie coefficients appearing 
in the theory of liquid crystals. Assuming in Eq. $9 \alpha_{1}=0$ and $\alpha_{5}=0$, we get the classical expression for the acoustic attenuation of the ordinary fluid related to viscosity only according the relation

$$
\alpha=\frac{2 \pi^{2} f}{\rho_{0} c^{3}}\left(\frac{4}{3} \eta+\eta_{V}\right)
$$

The third and the fourth terms in Eq. 9 are the additional terms of the acoustic attenuation which originate from the rotational motion of the clusters.
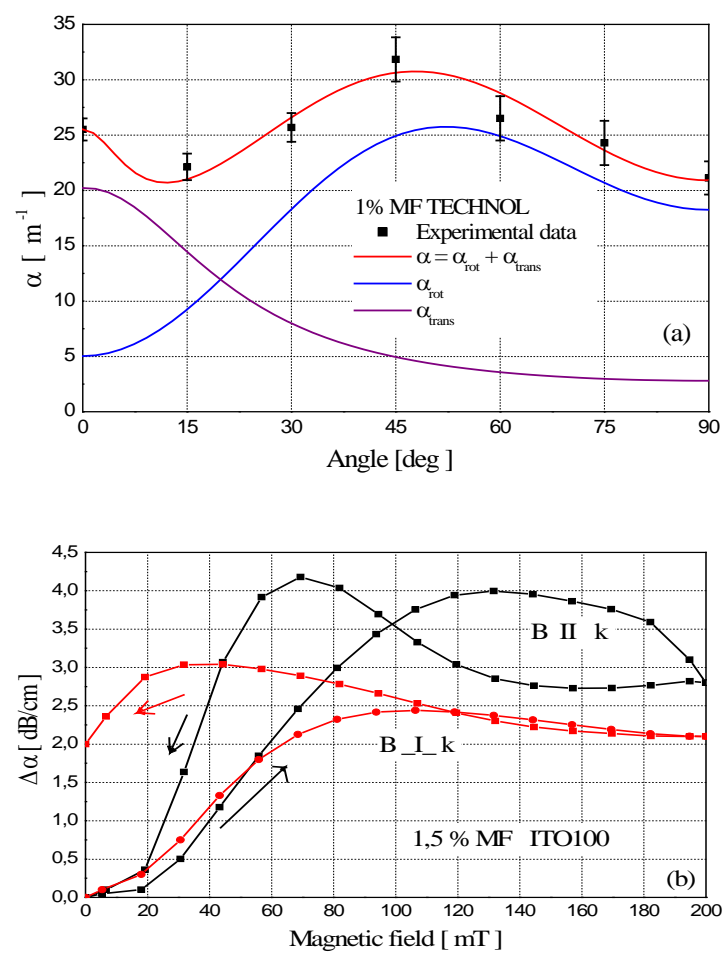

Fig. 5 (a) Anisotropy measurement of the acoustic wave attenuation $(f=13 \mathrm{MHz}, B=200 \mathrm{mT})$ in the MF based on TECHNOL and the components $\alpha_{\text {rot }}, \alpha_{\text {tr }}$ of the Taketomi functions [18] calculated for $20{ }^{\circ} \mathrm{C}$ and (b) comparison of changes of the attenuation in $1.5 \%$ MF based on ITO 100 ( $f=$ $5 \mathrm{MHz}, t=20{ }^{\circ} \mathrm{C}$ ) in magnetic field $\boldsymbol{B}$ perpendicular and parallel to wave vector $\boldsymbol{k}$, for constant increase rate $2,2 \mathrm{mT} / \mathrm{min}$

Table 1 Selected parameters $4 / 3 \eta_{\mathrm{S}}+\eta_{\mathrm{V}}, \alpha_{5}, \alpha_{1}, k$ and $r_{\mathrm{cl}}$ obtained from the fit of the function describing the measured anisotropy of the acoustic attenuation (Fig. 5a) [18] using the least-square method for temperature $20^{\circ} \mathrm{C}$.

\begin{tabular}{|l|l|l|l|l|}
\hline $\begin{array}{l}4 / 3 \eta_{\mathrm{S}}+\eta_{\mathrm{V}} \\
{\left[\mathrm{Nsm}^{-2}\right]}\end{array}$ & $\begin{array}{r}\alpha_{5} \\
{\left[\mathrm{Ns} \mathrm{m}^{-2}\right]}\end{array}$ & $\begin{array}{r}\alpha_{1} \\
{\left[\mathrm{Nsm}^{-2}\right]}\end{array}$ & $\begin{array}{r}\mathrm{k} \\
{\left[\mathrm{Nm}^{-1}\right]}\end{array}$ & $\begin{array}{r}\mathrm{r}_{\mathrm{cl}} \\
{[\mathrm{nm}]}\end{array}$ \\
\hline 0,0132 & 0,01448 & $-0,03854$ & 2,033 & 47,9 \\
\hline
\end{tabular}

The measurement of the acoustic attenuation as a function of the angle $\phi$ between the direction of propagation represented by $\boldsymbol{k}$ and that of the magnetic field $\boldsymbol{B}$ (anisotropy) for magnetic fields when the majority of the particles are involved in the cluster structures, confirmed the contribution of two components of the attenuation $\left(\alpha_{\text {rot }}+\alpha_{\mathrm{tr}}\right)[17,18]$ related to the rotational and translational degrees of freedom. It is possible also to estimate the per cent contribution of acoustic attenuation to the individual kinds of cluster motion.

\section{CONCLUSIONS}

In conclusion, the presented methods of acoustic spectroscopy applied to materials with nanostructures and/or nanoparticles evidently documents that the acoustic spectroscopy methods can be the powerful facility to obtain important information about investigated material properties. The MOS interfaces investigation offers the information about some their physical properties which can complete the data obtained using different, mostly electrical methods. The acoustic spectroscopy of magnetic fluids provides also very important information about structural properties under the various external conditions.

\section{ACKNOWLEDGMENTS}

The author would like to thank Mr. F. Černobila for technical assistance. This work was financially supported by the $R \& D$ operational program Centrum of excellence of power electronics systems and materials for their components I, II, No. OPVaV-2008/2.1/01-SORO, ITMS 2622012003 funded by European Community and projects VEGA 2/0077/09.

\section{REFERENCES}

[1] Physical Acoustics, Ed. By W. P. Mason and R. N. Thurston.: ACADEMIC PRESS, Vol. I - XI Series, $1964-1975$.

[2] MIDDlEMAN, S. - HOCHBERG, A. K.: Process Engineering Analysis in Semiconductor Device Fabrication, McGraw/Hill, Inc. (1993).

[3] KOBAYASHI, H. - ASUHA, A. - MAIDO, O. TAKAHASHI, M. - IWASA, M.: J. App. Phys. 94, 7328 (2003).

[4] ABBATE, A. - MAN, K. J. - OSTROVSKIJ, I. V. DAS, P.: Solid State Electronics 36, 697 (1993).

[5] BURY, P. - JAMNICKÝ, I. - ĎURČEK, J.: Phys. Status Solidi A 126, 151 (1991).

[6] BURY, P. - JAMNICKÝ, I.: Acta Phys. Slovaca 46, 693(1996).

[7] HOCKICKO, P. - BURY, P. - SIDOR, P. KOBAYASHI, H. - TAKAHASHI, M. - YANASE, T.: Cent. Eur. J. Phys. 9(1) (2011).

[8] RACKO, J. - VALENT, P. - BENKO, P. DONOVAL, D. - HARMATHA, L. - PINTEŠ, P. BREZA, J.: Solid State Electronics 52 (2008), 1755.

[9] BURY, P. - KOBAYASHI, H. - TAKAHASHI, M. - IMMAMURA, K. - SIDOR, P. - ČERNOBILA, F.: Cent. Eur. J. Phys. 7(2), 237(2009).

[10] ODENBACH, S.: Colloids and Surfaces A: Physicochem. Eng. Aspects, 217 (2003) 171.

[11] NAKATSUKA, K.: J. Magn. Magn. Mater. 122 (1993) 387.

[12] KOPČANSKÝ, P. - MARTON, K. KONERACKÁ, M. - TIMKO, M. - POTOČOVÁ, I. J.: Magn. Magn.Mater. 272-276, Part 3( 2004) 2377. 
[13] ROZYNEK, Z. et al.: Eur. Phys. J. E 34 (2011) 28.

[14] KÚDELČÍK, J. - BURY, P. - KOPČANSKÝ, P. TIMKO, M.: Physics Procedia 9 (2010) 78-81.

[15] JÓZEFCZAK, A. - SKUMIEL, A. - ŁABOWSKI, M.: J. Magn. Magn.Mater. 252 (2002) 356.

[16] VINOGRAGOV, A. N.: Colloid Journal, 65 (2003) 539.

[17] SKUMIEL, A.: J. Phys. D: Appl. Phys. 37 (2004) 3073.

[18] TAKETOMI, S.: J. Phys. Soc. Japan 55 (1986) 838.

[19] KÚDELČÍK, J. - BURY, P. - DRGA, J. KOPČANSKÝ, P. - ZÁVIŠOVÁ, V. - TIMKO, M.: Acta Physica Polonica A. Vol. 121, pp. 1170 (2012).

[20] KÚDELČÍK, J. - BURY, P. - DRGA, J. KOPČANSKÝ, P. - ZÁVIŠOVÁ, V. - TIMKO, M.: J.Magn. Magn. Mat. 326 (2013) 75.
Received November 30, 2012, accepted March 15, 2013

\section{BIOGRAPHY}

Peter Bury received the M.Sc. in experimental physics in 1972 and PhD. in 1982 at the Faculty of Natural Sciences (Faculty of Mathematics and Physics), Comenius University, Bratislava. Currently he is Professor of Physics of Condensed Matter and Acoustics at Department of Physics, Faculty of Electrical Engineering, Žilina University. The most of his research work was orientated on the study of semiconductors and semiconductor structures using acoustic methods. Formerly he investigated $\mathrm{Cr}$ states in GaAs using APR technique, later deep centres in both semiconductor and semiconductor structures using acoustic transient spectroscopy (A-DLTS). He used methods of acoustic spectroscopy also to study glasses, ferroelectrics and at present also magnetic fluids. 